\title{
Prevention of Diabetes in Nonobese Diabetic Mice by Dendritic Cell Transfer
}

\author{
Michael J. Clare-Salzler, Jacqueline Brooks, Andrew Chai, Katja Van Herle, and Claudette Anderson \\ Department of Medicine, University of California at Los Angeles, California 90024
}

\begin{abstract}
The purpose of this study was to determine the effect of dendritic cell (DC) transfers on the incidence of diabetes in female nonobese diabetic (NOD) mice. Groups of 4-wk-old NOD female mice were given a single foot pad of DCs (70-90\% purity) isolated from the draining lymph nodes (LN) of the pancreas (PLN), the cervical LNs, or the axillary/inguinal LNs. In addition, other groups of NOD mice received purified spleen DCs, purified PLN T cells (the major contaminating population in DC preparations), or the injection vehicle PBS. All groups were monitored for diabetes for one year.

Significant protection from diabetes was observed in NOD mice receiving $>1 \times 10^{4}$ PLN DCs in comparison to mice receiving other DCs populations, PLN T cells, or PBS ( $P$ $<0.05)$. The pancreata of NOD mice that received PLN DCs demonstrated significantly lower levels of lymphocytic infiltration in the islets that age-sex matched nondiabetic female NOD control mice $(P<0.05)$. LN cells from nondiabetic NOD mice that received PLN DC protected irradiated female recipients from the adoptive transfer of diabetes to a greater degree than LN cells from age and sex matched nondiabetic female NOD mice that did not receive PLN DC transfers at $36 \mathrm{~d}(P=0.014)$ and at $1 \mathrm{yr}(P=0.0015)$ after transfer.

These data suggest that the PLN DC transfers are able to modulate autoimmunity and limit diabetes expression in the NOD mouse. PLN DCs transfers may regulate autoimmunity by the induction of regulatory cells. (J. Clin. Invest. 1992. 90:741-748.) Key words: nonobese diabetic mice - dendritic cells $\bullet$ diabetes $\bullet$ autoimmunity
\end{abstract}

\section{Introduction}

The nonobese diabetic (NOD) ${ }^{1}$ mouse is a well established model of type 1 diabetes (1-4). NOD mice universally develop insulitis but not all animals develop diabetes (5). Approximately $25 \%$ of NOD/Ym female mice and $70 \%$ of male mice do not develop diabetes (UCLA colony data). A reduction in the incidence of diabetes in NOD mice has been accomplished by a number of nonspecific manipulations including injection

Address reprint requests to Dr. Michael Clare-Salzler, Room 46$118 \mathrm{CHS}$, Department of Medicine, Division of Endocrinology, University of California Los Angeles, CA 90024.

Received for publication 12 November 1990 and in revised form 2 March 1992.

1. Abbreviations used in this paper: A/I, axillary/inguinal; DC, dendritic cell; LN, lymph nodes; NOD, nonobese diabetic; PLN, pancreatic LN; SMG, submandibular gland; SMLR, syngenetic mixed lymphocyte response.

J. Clin. Invest.

(C) The American Society for Clinical Investigation, Inc.

0021-9738/92/09/0741/08 $\$ 2.00$

Volume 90, September 1992, 741-748 of CFA, viruses, and the cytokines IL-1, IL-2, and tumor necrosis factor (TNF) (6-9). Investigators have suggested that the NOD mouse contains cells that function to inhibit the autoimmune response which may be enhanced by these maneuvers $(6,8,10)$. For example, natural suppressor cells can be augmented in NOD mice by the administration of CFA (6). Other studies have suggested that protective cells are CD4-positive $T$ cells that are found in unmanipulated NOD mice, to varying degrees at different ages (10).

The "honeymoon period," an apparent spontaneous remission of type 1 diabetes seen in some new onset patients may represent a down modulation of the autoimmune response for a brief period of time. Peripheral blood leukocyte analysis from prediabetic patients suggests that there are increased numbers of cells with the suppressor/inducer phenotype before diabetes onset and the number of these cells diminishes at the time of diabetes onset (11). Combined, these animal and human studies suggest that the autoimmune response to the beta cell can be modulated, perhaps through regulatory cells.

Regulatory or effector $\mathrm{T}$ cells responses may be induced by stimulation with antigen-presenting cells. The dendritic cell (DC), a unique antigen-presenting cell, may play an important role in the regulation of the autoimmune response. Various investigators have demonstrated that DCs are able to transfer experimental autoimmune diseases such as thyroiditis and encephalomyelitis with great efficiency (12-14). Conversely, DCs also have the potential to control the autoimmune response by a variety of mechanisms. DCs are the chief stimulators of the syngeneic mixed lymphocyte response (SMLR) (15). The SMLR, which is depressed in NOD mice (16), may be important in the generation of suppressor/inducer cells (17). Furthermore DCs pulsed with a high concentration of an antigen can inhibit the immune response to that antigen (18). In contrast pulsing with low antigen concentrations stimulates the immune response (18). DCs also participate in the thymic selection of the $T$ cell repertoire (19). In addition, these antigen-presenting cells may play a role in the shaping of peripheral tolerance (20). Thus DCs have the potential to modify the autoimmune response.

Little is known about the function of DCs in NOD mice. As discussed DCs could function to either augment or reduce the autoimmune response of NOD mice. We chose to investigate the function of DCs in the NOD mouse in transfer experiments. We were particularly interested in ascertaining the function of pancreatic lymph node (PLN) DCs for the following reasons. First, DCs are an early component of the islet infiltrate in the animal models of type 1 diabetes $(21,22)$. In addition, DCs have been shown to take up antigen in tissues and travel via the afferent lymphatics to the draining LNs where they stimulate immune responses $(23,24)$. Thus DCs in the islets may acquire relevant antigens and migrate to the draining PLN where they stimulate an immune response. The following experiments demonstrate the effect of PLN DC transfers on diabetes incidence in NOD mice. 


\section{Methods}

Animals. NOD/Ym mice of various ages were obtained from the UCLA colony. NOD mice used as DCs donors were female, 8-20 wk of age. Recipient mice were 4-wk-old NOD females. The colony at UCLA is checked yearly, and was negative for a number of viruses and mycoplasma currently, and during the period of experimentation. The viruses screened for include: mousepox, murine hepatitis, polyoma, Sendai, Reovirus 3, adenovirus, Theller's GD-VII, LCMV, K virus, and minute virus. In addition, the skin, oral cavity, rectal area, genito-urinary area, and abdominal cavity were all inspected for the presence of infection or tumors before use. If infection or tumors were found the animals were excluded from experiments. Mice were also evaluated for diabetes by urine glucose testing at the time of use. NOD mice with glycosuria were also excluded. 4-wk-old female littermates served as recipients. These littermates were randomly divided amongst the various experimental groups to control for potential variation in the incidence of diabetes amongst different litters and also controlled for the vertical transmission of viruses. The different experimental groups of recipient mice were housed two to three animals per microisolator in close proximity to each other and fed standard lab chow and water from the same sources ad lib. All animals were handled by the same animal care technicians.

Monitoring for diabetes. The urine of recipient mice was monitored weekly for glucose with Tes-Tape (Eli Lilly \& Co., Indianapolis, IN) after injection. Mice that were found to be glucose positive for three consecutive days were then placed on insulin therapy with Lente insulin (Eli Lilly \& Co.) and monitored daily for glycosuria. Recipients that were urine glucose negative at the time of sacrifice were tested for hyperglycemia by capillary blood glucose with an Accucheck II (Boehringer Mannheim GmbH, Mannheim, Germany). Glucose values of 60 to $140 \mathrm{mg} / \mathrm{dl}$ were considered to be normal. Diabetes was defined as glucose values above $300 \mathrm{mg} / \mathrm{dl}$. The incidence of diabetes was closely monitored in the colony mice over the period of the experiment.

DC purification. DCs were purified from PLN, axillary/inguinal (A/I) LNs, and cervical LNs, as described by Knight (13). Spleen DCs were purified by culturing freshly isolated NOD whole spleen cells at a concentration of $1 \times 10^{7} / \mathrm{ml}$ overnight in RPMI 1640 plus 2-ME as described (13). Cells were washed one time in medium and then separated as before on metrizamide (Sigma Chemical Co., St. Louis, MO) gradients. This preparation consistently yielded DCs from all lymphoid tissues that were $70-90 \%$ pure. Purified DCs were then cells washed twice in PBS and used for injection. PLN DCs were obtained in very small numbers. 8-12 (8-20-wk-old) NOD female mice yielded $5.0 \times$ $10^{4}-3.0 \times 10^{5}$ PLN DCs. The different groups of DCs (i.e., PLN DCs, cervical DCs, etc.) given to recipient mice were derived from the same pool of NOD donors to control for possible variation in donor cells and to control for the transmission of viruses to recipients.

$T$ cell preparation. $T$ cells were prepared by adherence to nylon wool columns as described by Julius (25). The purity of the preparation was assessed by labeling cells with Thy 1.2 (Becton-Dickinson Co., Mountain View, CA). This method consistently yielded T cells of 80 $90 \%$ purity.

Foot pad injection. Cells were suspended in PBS. 4-wk-old NOD female mice were then lightly anesthetized with ether, and injected in each hind foot pad with $25 \mu \mathrm{l}$ of various doses of DCs $\left(1.0 \times 10^{4}-2.0\right.$ $\left.\times 10^{5} \mathrm{DCs}\right)$, $\mathrm{T}$ cells $\left(1.5 \times 10^{4}\right)$ from the various lymphoid tissues, or PBS.

$D C$ migration studies. The migration of DCs injected into the foot pad of NOD mice was studied by labeling these cells with a vital fluorescent dye, Hoechst 33342 (Sigma Chemical Co.), which labels cell nuclei. DCs were exposed to Hoechst $33342(6 \mu \mathrm{g} / \mathrm{ml})$ for $15 \mathrm{~min}$ at $37^{\circ} \mathrm{C}$. The cells were then washed twice with PBS before injection. DC labeling was assessed with a Nikon Optiphot microscope with fluorescent capability prior to injection. DC viability was assessed for up to $8 \mathrm{~h}$ after labeling with trypan blue exclusion and was $90 \%$ or greater. 4-wkold female NOD mice were injected in the foot pad with $1 \times 10^{5}$ labeled PLN DCs or A/I DCs. Popliteal LNs, inguinal LNs, cervical LNs, axillary LNs, spleen, thymus, and pancreas were harvested at 3 and $5 \mathrm{~d}$ after injection and $5 \mu \mathrm{m}$ frozen tissue sections prepared. These sections were then examined with fluorescent microscopy. 4-wk-old control mice (BALB/c and B10.BR) were also examined in a similar fashion.

Popliteal LN proliferative assay. Popliteal LNs of NOD mice were removed $5 \mathrm{~d}$ after the injection of various populations of purified DCs. NOD mice were injected in one footpad with $5 \times 10^{4}$ PLN DCs and in the other footpad with PBS. Control NOD mice were injected in a similar fashion with PBS and $5 \times 10^{4}$ axillary/inguinal LN DC in the other footpad. Single cell suspensions of the popliteal LN were prepared by teasing it over a steel sieve. 100,000 cells were placed in RPMI $164010 \%$ FCS, plus 2ME, counted, placed in 96-well culture plates, and pulsed with $1 \mu \mathrm{Ci}\left[{ }^{3} \mathrm{H}\right.$ ]thymidine for $16 \mathrm{~h}$ before harvesting. All experiments were performed in triplicate.

Histology. NOD pancreata were removed at sacrifice. The pancreata were fixed in $10 \%$ buffered formalin, processed, and paraffin sections examined. Five interrupted serial sections cut $150 \mu \mathrm{m}$ apart were stained for insulin by indirect immunohistochemistry, and then counterstained with hematoxylin. The five sections were scored blindly by an independent observer. The data obtained were analyzed by a second blinded observer. The scoring system employed used a 1 to 10 point scale illustrated in Fig. 1. In addition the number of islets in each section was counted and the total for five sections was averaged.

Diabetes transfer protocol. The protocol used for the transfer of diabetes was that described by Wicker et al. (4). Briefly, 8-11-wk-old NOD female mice were irradiated ( 850 rads) and then given $2.0 \times 10^{7}$ spleen cells by tail vein injection from an acutely diabetic NOD female (diabetes duration $<30 \mathrm{~d}$ ). The mice were then observed daily for the development of diabetes by urine glucose monitoring. Mice that were glycosuric were then tested for blood glucose by chemstrip. Diabetic mice demonstrated glucose values $>300 \mathrm{mg} / \mathrm{dl}$.

Inhibition of diabetes transfer. A modified 2-d transfer protocol was used to test the ability of cell populations to inhibit the transfer of diabetes. This protocol was similar to that described by Boitard et al. (10). Briefly, mice were irradiated as before and on day one given intravenous injections of either $2.0 \times 10^{7} \mathrm{LN}$ cells from 1-yr-old nondiabetic NOD mice that had received PLN DC transfers. The LN cells were pooled from the cervical, axillary, inguinal, PLN, and peri-aortic LNs. Other recipients received the same number of $\mathrm{LN}$ cells from donors that were age- and sex-matched NOD mice that did not receive PLN DC transfers. On the second day both groups received injections of $2.0 \times 10^{7}$ spleen cells from acutely diabetic NOD mice. In addition in each of the 2-d transfers an internal control group received the same dose of diabetic spleen cells alone.

\section{Results}

DC migration after footpad injection. The migration of DCs was assessed after labeling these cells with a nuclear vital fluorescein dye. These labeled cells, purified from the PLNs of 12-wkold NOD female mice, were injected into the footpad of 4-wkold NOD female mice. The labeled DCs were found only in the popliteal LN when examined on day 3 and 5 after injection. Fluorescent cells were not found in the pancreas, spleen, thymus, or other LNs. A few fluorescent cells were found in the footpad of injected mice. The vast majority of fluorescent cells were found in the subcapsular space and the medullary cords of the popliteal LN. No fluorescent cells were found in the efferent lymphatics. NOD mice injected with A/I DCs demonstrated the same pattern of migration. Control mice similarly injected in the footpad with DCs demonstrated the same findings. The fluorescent dye intensity diminished to low levels when LNs were examined at $7 d$ and was absent at day 14 . Thus analysis at later time points was prevented.

These experiments demonstrated that PLN and A/I DCs 

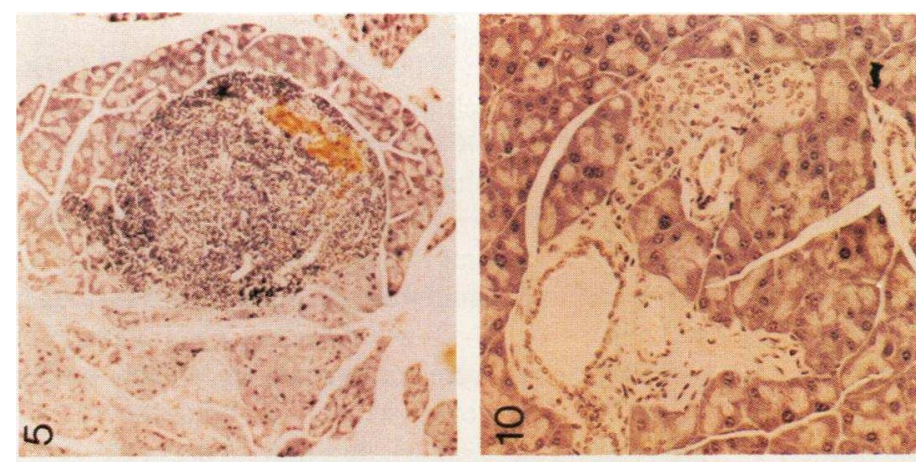

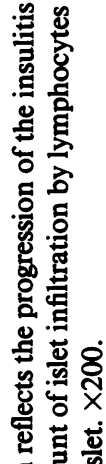
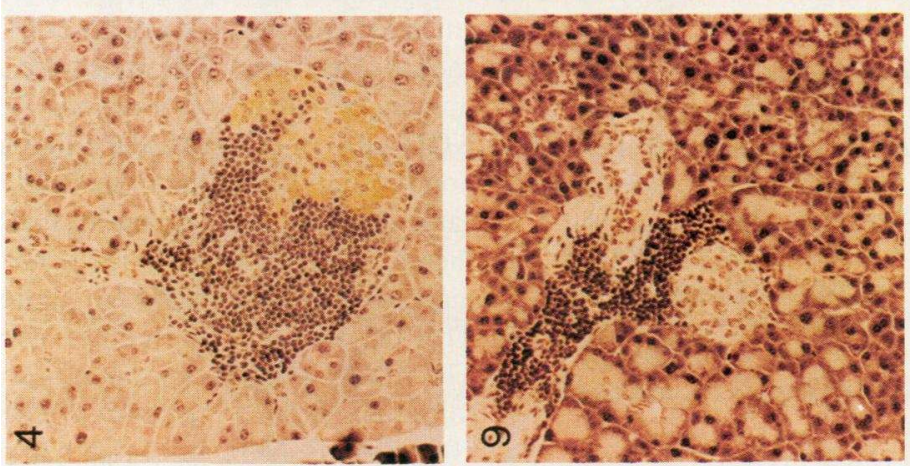

娄
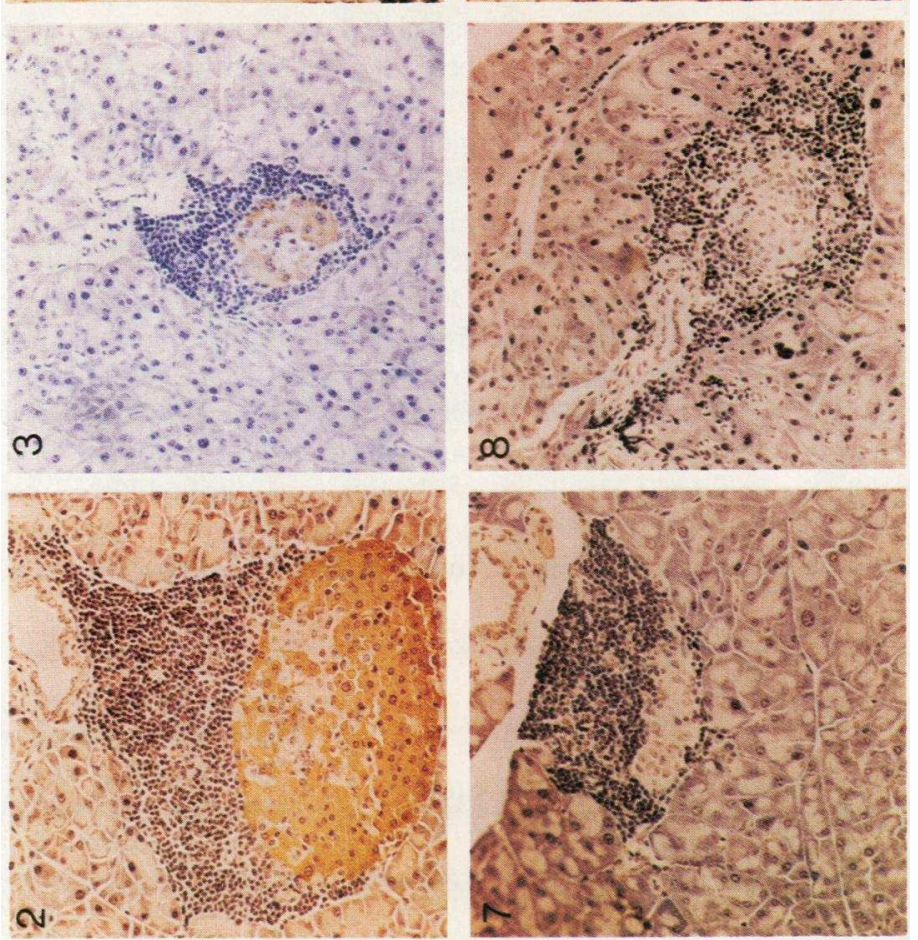

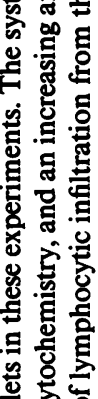

究

을.

.

ริ

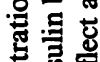

产象

帝

잉

家客

믈

政

急

$+2$

总要

$\frac{2}{3} \div$

a.

娄

a 80

.

通

运

言岕
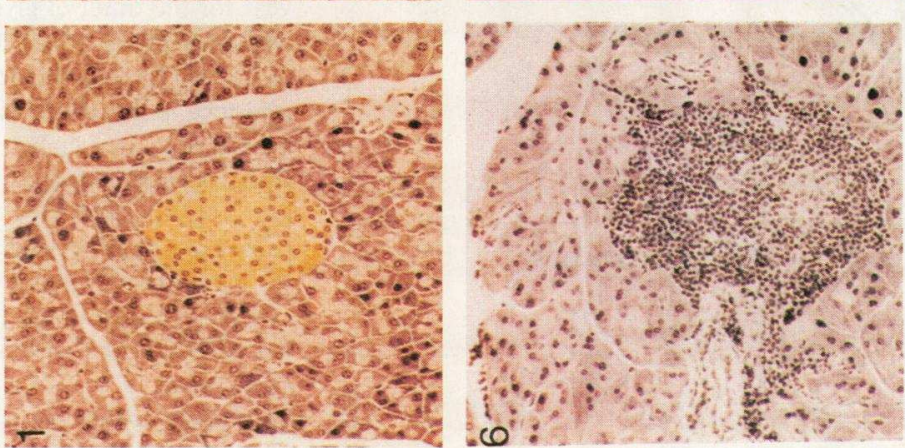

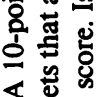

$<\frac{2}{2}$

造

会范

造。递

远

通

密苋 
Table I. Popliteal Lymph Node Proliferation Assay

\begin{tabular}{cccc}
\hline & \multicolumn{3}{c}{ Average cpm $\left[{ }^{3} \mathrm{H}\right]$ thymidine } \\
\cline { 2 - 4 } $\begin{array}{c}\text { Recipient } \\
\text { No. }\end{array}$ & PBS & A/I DCs & PLN DCs \\
\hline 1 & 350 & 495 & - \\
2 & 538 & 650 & - \\
3 & 268 & 261 & - \\
4 & 207 & 191 & - \\
5 & 421 & - & 1,440 \\
6 & 357 & - & 1,269 \\
7 & 533 & - & 1,870 \\
Average & $313.2 \pm 156$ & $399.2 \pm 211$ & $1,526.3 \pm 309^{*}$
\end{tabular}

4-wk-old NOD female mice received footpad injections of PBS in the left footpad, and either $5 \times 10^{4} \mathrm{~A} / \mathrm{I}$ DCs or PLN DCs in the right footpad on the same day. The animals were killed at day 5 after injection and $1 \times 10^{5}$ cells were placed in microtiter wells, pulsed with $1 \mu \mathrm{Ci}$ of $\left[{ }^{3} \mathrm{H}\right]$ thymidine, and harvested $16 \mathrm{~h}$ later. Each assay was performed in triplicate. The proliferative activity of the popliteal LN on the side that had received PLN DCs was significantly greater that of popliteal LNs that had been exposed to either PBS or A/I DCs $\left({ }^{*} P\right.$ $<0.05$ ). There was essentially no difference between the PBS and $\mathrm{A} / \mathrm{I}$ DC groups at the 0.05 significance level. Parametric analysis of variance was performed using the $t$ test.

from the NOD mouse migrate to the popliteal LN, and cannot be detected in other lymphoid tissue or the pancreas. Fluorescent DCs were seen in large numbers in these $\mathrm{LN}$. This suggests that the generation of an immune response by transferred DCs would likely be confined to the popliteal LN, at least initially.

Popliteal LN proliferative assays. The proliferative activity of cells from the popliteal LNs of 4-wk-old NOD mice, previously injected in the footpad with different populations of DCs from the same pool of donors, was assessed after overnight incubation with $\left[{ }^{3} \mathrm{H}\right.$ ] thymidine. Popliteal LN cells harvested 5 $\mathrm{d}$ after the injection of PLN DCs demonstrated significantly higher levels of proliferative activity than the popliteal LN cells that had received either PBS or A/I LN DCs $(P<0.001)$ (see Table I). The level of proliferative activity of popliteal LN cells that had been exposed to A/I LN DC or PBS were essentially equal.

These data suggest that PLN DCs are capable of producing cell proliferation in the popliteal $\mathrm{LN}$ to a greater degree than DCs isolated from LNs that are not associated with the lymphatic drainage of the pancreas.

$D C$ transfers. 4-wk-old NOD female mice were given bilateral footpad injections of pooled DCs (8-12 NOD mice) isolated from the spleens and various LNs of NOD mice that were 8-20 wk of age. DCs populations used in transfer experiments were consistently of $70-90 \%$ purity. The remaining cells were composed of $\mathrm{T}$ lymphocytes (10-20\%), B lymphocytes $(<5 \%)$, and macrophages (5-10\%) (data not shown). Recipient mice received various doses of DC $\left(1 \times 10^{4}\right.$ to $2 \times 10^{5} /$ mouse (see Fig. 2). The mice were then observed for the development of diabetes for one year. There was a $100 \%$ survival of NOD mice in experimental and control groups that remained nondiabetic during the observation period. Once animals became diabetic, survival was limited to a few weeks with insulin therapy. During the period of experimentation the incidence of diabetes in 1-yr-old NOD female colony mice was $\sim 75-78 \%$. This percentage has remained consistent over several years. The results of these experiments are displayed in Fig. 2. NOD mice that received injections of PBS developed diabetes in 7 of 10 recipients. NOD mice that had received DCs from LNs not associated with the pancreas, i.e., the $\mathrm{A} / \mathrm{I} \mathrm{LN}$, developed diabetes at a rate identical to that of the colony (78\%).

This was also true for the cervical LN DC population. Cervical LNs are the draining LN of the submandibular gland (SMG). The SMG of NOD mouse is also involved in an inflammatory process (sialitis). The sialitis process occurs concurrently the insulitis process of the pancreas (21). Cervical LN DCs were given to recipients to test the hypothesis that DC from a draining LN of an inflamed gland other than the pancreas were equally as effective in preventing diabetes. Cervical DCs however were found to provide no protection from diabetes $(8: 10 ; 80 \%$ diabetes $)$.

Nylon wool-purified PLN T cells were also given to recipients in a dose that was equivalent to the number of $\mathrm{T}$ cells found in PLN DC preparations $\left(1.5 \times 10^{4}\right.$ cells $)$. NOD mice

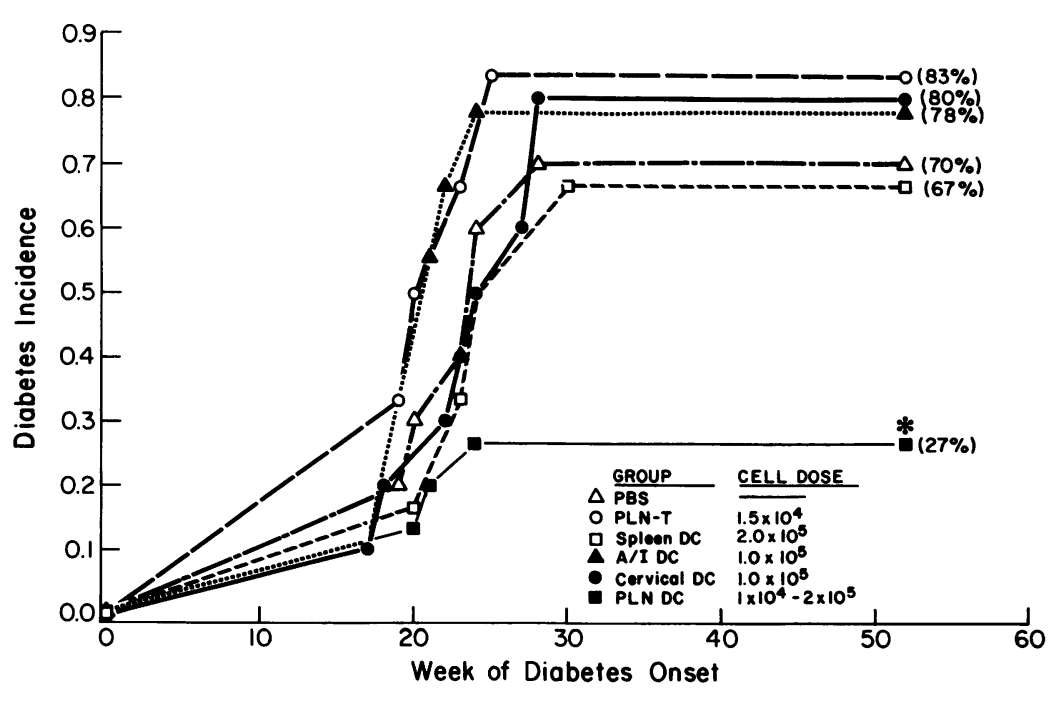

Figure 2. 4-wk-old NOD female mice received bilateral footpad injections of various DC populations (70-90\% purity) isolated from LNs and spleens of nondiabetic 8-20-wk-old NOD female mice. Other NOD mice received nylon wool-purified PLN T cells, and another group received the injection vehicle PBS. The mice were checked weekly for a period of one year for the onset of glycosuria by tes-tape. If mice were found to be glycosuric diabetes was confirmed by chemstrip. The incidence of diabetes in each group is plotted in this figure as a function of time (weeks). The incidence of diabetes in NOD mice that received PLN DCs was statistically significant from all other groups $(P<0.05)$ with the exception of mice that received spleen DC transfers $(P=0.14)$. If, however, the three animals that received low doses of PLN DCs are removed from the analysis the difference between the PLN DC and spleen DC group reaches statistical significance $(P=0.01)$. Survival curve analysis of diabetes incidence using the log rank test was performed to statistically analyze the data. 
receiving PLN T cells developed diabetes in 5:6 (83\%) of recipients.

In marked contrast PLN DCs transfers were found to be highly protective in that only $4: 15$ animals were observed to develop diabetes in a 1-yr period. Two of the four animals that became diabetic in this group received low doses of PLN DCs $\left(1 \times 10^{4}\right.$ cells/recipient $)$. If the three animals that received 1 $\times 10^{4}$ PLN DCs are removed from the analysis then the diabetes incidence in this group drops to $17 \%$. The difference in diabetes incidence between the PLN DC group (including those animals that received low doses of PLN DCs) and the other transfer groups reaches statistical significance $(P<0.05)$ in all cases with the exception of the spleen DC group ( $P$ $=0.13)$. NOD mice receiving spleen DCs, which were given at higher doses $\left(2 \times 10^{5}\right.$ cells/recipient $)$, however did not markedly effect the incidence of diabetes in recipients $(4: 6 ; 67 \%)$. If the three animals receiving $1 \times 10^{4}$ PLN DCs are removed from the analysis the difference between the spleen DC group and the PLN DC group also reaches statistical significance ( $P$ $=0.01$ ).

These results demonstrate that PLN DCs given to 4-wk-old NOD female mice as a single footpad injection markedly inhibit the development of diabetes in the recipient. This protective effect is not seen to any degree in the other populations DCs or with PLN T cells.

Pancreatic histology of NOD mice. The effect of PLN DC transfers on the insulitis process of NOD mouse recipients was assessed by histologic examination of the pancreas. In a pilot study four female NOD mice that received PLN DCs at $4 \mathrm{wk}$ of age and four female NOD mice that received PBS injections at the same age were killed at $8 \mathrm{wk}$ of age and their pancreata examined. The NOD mice that received PLN DCs did not have any evidence of lymphocytic infiltration in the islets examined, whereas the PBS injected mice all had evidence of insulitis at this age (data not shown).

In the follow up study the pancreata of NOD mice that had received either PLN DCs or A/I DCs at 4 wk of age, and then became diabetic, were evaluated histologically within 4-8 wk of the onset of their disease (see Table II). In addition, the pancreata of untreated female NOD colony mice were also examined within 4-6 wk of the onset of their diabetes. The islets of all of the diabetic NOD groups (including those that had received PLN DCs) were infiltrated with a large number of lymphocytes and were small in size in comparison to those of the nondiabetic group (data not shown). The average infiltrate score for these diabetic animals were similar (range; 8.1-9.2). Very few islets in the pancreas of the diabetic groups were positive for insulin by immunohistochemistry. The pancreata of NOD mice that had received PLN DCs demonstrated a histologic score similar to that of the other diabetic mice examined. The PLN DC recipients however demonstrated more islets per section (average 15.3 per five sections). This difference, however, did not reach statistical significance.

The pancreata of NOD that had received PLN DCs at $4 \mathrm{wk}$ of age, but failed to develop clinical diabetes, were examined when the animals reached 1 yr of age or greater. In addition, the pancreata of age-sex matched colony NOD mice that failed to develop diabetes were also examined. The histologic appearance of the islets of both of the nondiabetic groups were markedly different from their diabetic counterparts. As seen in Table II, the number of surviving islets was much greater and the infiltration scores were far lower than those of diabetic animals.
Table II. Pancreatic Histology Scores of NOD Mice

\begin{tabular}{llrcc}
\hline \multicolumn{1}{c}{ NOD group } & $\begin{array}{c}\text { Cells } \\
\text { transferred }\end{array}$ & \multicolumn{1}{c}{$n$} & \multicolumn{1}{c}{$\begin{array}{c}\text { Average } \\
\text { score }\end{array}$} & $\begin{array}{c}\text { Average islet no./ } \\
\text { five sections }\end{array}$ \\
\hline Diabetic NOD & none & 6 & $8.8 \pm 1.2$ & $5.6 \pm 5.2$ \\
Diabetic NOD & A/I DCs & 6 & $9.2 \pm 1.0$ & $9.8 \pm 8.2$ \\
Diabetic NOD & PLN DCs & 6 & $8.1 \pm 1.4$ & $15.3 \pm 5.8$ \\
Nondiabetic NOD & None & 11 & $4.2 \pm 2.2$ & $51.9 \pm 42.7$ \\
Nondiabetic NOD & PLN DCs & 12 & $2.3 \pm 0.9^{*}$ & $50.0 \pm 21.2$
\end{tabular}

The pancreata of diabetic female NOD mice that had received no treatment (spontaneous colony diabetes), and NOD mice that developed diabetes after receiving either NOD PLN or A/I DCs at 4 wk of age were examined histologically in paraffin sections stained with hematoxylin, and for insulin by immunocytochemistry within 4-8 wk of disease onset. In addition the pancreata of nondiabetic 1-yr-old NOD PLN DC recipients, and nondiabetic age-sex matched colony mice, were examined in a similar fashion. The pancreata of all mice were scored blindly using a 10-point scale (see Fig. 1). Untreated diabetic NOD mice had few islets that were negative for insulin. Diabetic NOD mice that had received PLN DCs had greater numbers of islets/five sections (15.3) than did the other two diabetic NOD groups. The colony nondiabetic NOD mice had markedly lower infiltrate scores and many large insulin-positive islets. NOD mice that received PLN DCs had significantly lower infiltration scores than those of colony nondiabetic animals $(* P<0.05)$. Results are expressed as an average $\pm \mathrm{SD}$. Analysis of variance was performed using the post hoc $t$ test.

The NOD mice that received PLN DCs demonstrated significantly lower infiltration scores than those of the nondiabetic NOD mice that did not receive PLN DC transfers $(P<0.05 ; t$ test). The islets of NOD mice that received PLN DC transfers were often completely free of infiltration. If lymphocytic accumulation did occur, it remained outside of the islet in the periductal region.

Inhibition of diabetes transfer. The possibility that cell-mediated suppression of insulitis and diabetes is enhanced in NOD mice that received PLN DCs was assessed in a modified adoptive transfer setting. $100 \%$ of NOD female recipients that were irradiated and then injected with diabetic spleen cells $48 \mathrm{~h}$ later developed diabetes within $22 \mathrm{~d}$ of transfer (see Fig. 3). To test for the inhibition of diabetes transfer $L N$ cells ( pooled from the cervical, A/I, PLN, peri-aortic LN, and popliteal LN) from NOD mice were given to irradiated NOD female recipients on day one, followed by diabetic spleen cells on day two. LN cells from 1-yr-old nondiabetic female NOD mice that did not receive DC transfers initially inhibited the transfer of diabetes in some animals (5:8 [63\%] animals became diabetic at $22 \mathrm{~d}$ ). This protection, however, was short lived in that 8:8 recipients became diabetic within $36 \mathrm{~d}$ of transfer (see Fig. 3).

When irradiated NOD mice were injected with pooled LN cells from nondiabetic female NOD mice that received PLN DC on day one the transfer of diabetes was markedly inhibited. $31 \%(5: 16)$ of NOD recipients became diabetic within $22 \mathrm{~d}$ and only $50 \%$ (8:16) of mice became diabetic by $36 \mathrm{~d}$ after transfer. The five mice that eventually became diabetic did so at 50,78 , 100,121 , and $181 \mathrm{~d}$ after transfer. Three mice (19\%) remained nondiabetic at $1 \mathrm{yr}$ of age. The protection seen in this group at $36 \mathrm{~d}$ was statistically different from the other two groups ( $P$ $=0.014$ ). Furthermore survival curve analysis of the diabetes incidence using the log rank test indicated that NOD mice that 


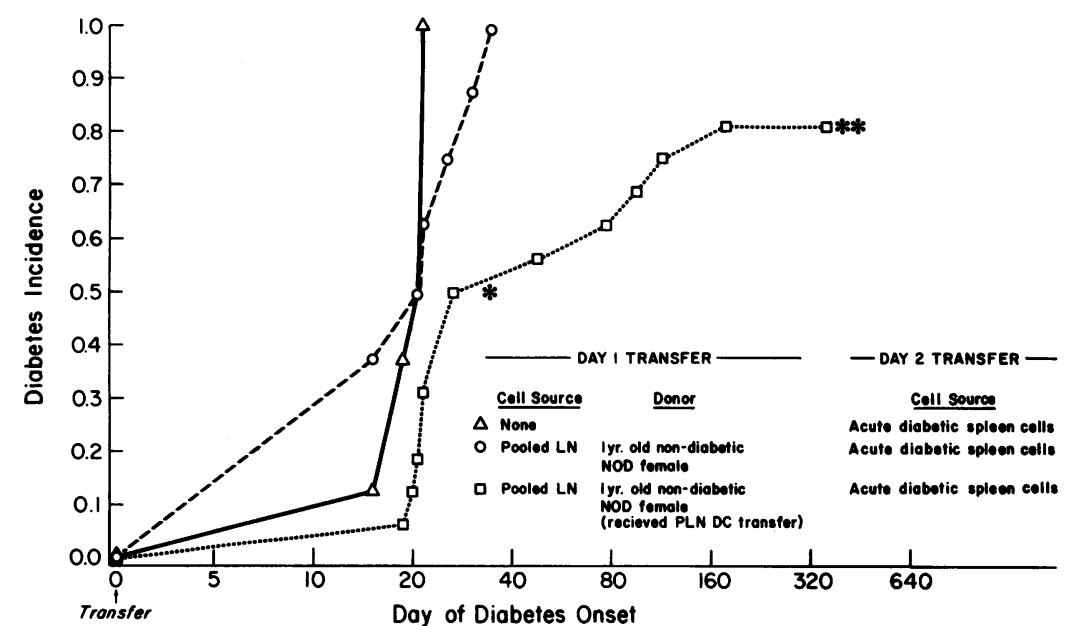

Figure 3. NOD recipients were 8-11-wk-old NOD females that received 850 rads. On day 1 the mice received $2.0 \times 10^{7}$ cells i.v. of the designated cell type. On day $22.0 \times 10^{7}$ diabetic spleen cells were given. Urine sugars were monitored daily. Mice that received LN cells from NOD mice that were recipients of PLN DC transfers showed a significant reduction in the incidence of diabetes at day 36 after transfer $\left({ }^{*} P\right.$ $=0.014$; chi square test) in comparison to the other two groups. Furthermore survival curve analysis of diabetes incidence using the log rank test demonstrated a highly significant difference between the NOD mice that received LN cells from NOD mice that received $\mathrm{LN}$ cells from NOD mice that received PLN DC transfers and the other groups $\left({ }^{* *} P\right.$ $=0.0015)$. received PLN DC transfer were protected to a significantly greater degree that animals from the other two transfer groups $(P=0.0015)$.

These experiments suggests that NOD mice that received PLN DC transfers contain cellular elements that inhibit the transfer of diabetes to a greater degree than $\mathrm{LN}$ cells from agematched nondiabetic control NOD mice. There was, however, an initial delay in the transfer of diabetes in some animals that received LN cells from 1-yr-old unmanipulated NOD mice given on day one. 1-yr-old nondiabetic mice have a low probability of developing spontaneous diabetes and may contain regulatory elements that inhibit the development of the disease in these animals. It may be that PLN DC transfers enhance similar regulatory elements in transferred animals.

\section{Discussion}

In these studies we demonstrate that PLN DC transfers markedly perturb the autoimmune response of prediabetic NOD mice and prevent diabetes in the majority of recipient animals. PLN DCs, when compared to DCs isolated from other LN groups, were unique in their ability to protect recipients from diabetes. Histologic examination of the islets of 1 -yr-old nondiabetic NOD mice that received PLN DCs demonstrated a low degree of lymphocytic infiltration suggesting that a single transfer of PLN DCs was able to inhibit, but not completely ablate, the autoimmune process. Furthermore, $\mathrm{LN}$ cells from NOD mice that received PLN DC transfers markedly inhibited the adoptive transfer of diabetes by diabetic spleen cells demonstrating that these recipients contained potent regulatory cells. These studies suggest that PLN DCs may play an important role in the regulation of autoimmunity.

Of particular interest was the finding that isolated PLN DCs in comparison to other populations of NOD LN DCs were highly effective at modulating the autoimmune response. There are a number of potential explanations for the unique protective effect provided by PLN DC transfers. First, cytokines such as IL-1 or granulocyte macrophage-colony-stimulating factor are known to augment DC function. It could be envisaged that PLN DCs are "activated" upon exposure to these cytokines produced in the inflamed islet $(27,28)$. After cytokine exposure DCs may become more potent simulators of regulatory cells. DCs isolated from the cervical $\mathrm{LN}$, an $\mathrm{LN}$ associated with the inflammation of the SMG of NOD mice
(26), however, were not protective. This suggests that the protective effect of PLN DCs is not due to their exposure to an inflammatory process.

Second, the protective effect seen with PLN DC transfers may be due to the acquisition of islet antigen(s) by these antigen-presenting cells. DCs have been shown to take up antigen in tissue sites and migrate via the afferent lymphatics to the draining $L N$ where they activate $T$ and $B$ lymphocytes $(23,24$, 34 ). We postulate that DCs present in the insulitis lesion (21, 22 ) acquire islet antigen(s) before they migrate to the PLN. This hypothesis is supported by the observation that DCs isolated from LNs that do not share a common lymphatic drainage with the pancreas, i.e., cervical LNs and A/I LNs, were not protective. Furthermore, preliminary experiments demonstrated that the transfer of A/I LN DCs, which do not provide protection in the transfer setting, do so if they are pulsed with sonicated islets before their transfer (unpublished data). Finally, a preliminary analysis of the histopathology of the SMG of NOD mice that received cervical LN DCs transfers demonstrated low levels of lymphocytic infiltration in this organ in comparison to the SMG of NOD mice that received PLN or A/I LN DC transfers. This suggests that cervical LN DC transfers modify the immune response in the SMG. These results support the hypothesis that the suppression of disease by DC transfers is dependent on their exposure to tissue antigens and on this basis may be tissue- or antigen-specific.

Because DCs are potent antigen-presenting cells for $T$ cell responses we hypothesized that the protection provided by PLN DC transfers may be due to the stimulation of regulatory cells. Indeed, we found that LN cells from NOD mice that received PLN DC transfers inhibited the transfer of disease by diabetic spleen cells to a significantly greater degree than did LN cells of unmanipulated nondiabetic NOD mice. The ability of DCs to stimulate regulatory cells may be related to their potency as stimulators of the SMLR, a response in which suppressor/inducer cells are produced $(29,30)$. We have found that the DCs of NOD mice, like those of nonautoimmune strains, are potent stimulators of the SMLR (manuscript in preparation). It is possible that PLN DCs injected into the footpad effectively stimulate suppressor/inducer cells in the popliteal LN that may be specific for the insulitis process. Lymphocytes primed in the popliteal LN by PLN DCs may then exit this tissue and home to sites of inflammation such as the islet or other lymphoid compartments. In support of this con- 
cept Inaba et al. recently demonstrated that lymphocytes primed by antigen pulsed DC disseminated to other lymphoid compartments (31).

Previous studies support the hypothesis that DCs play a key role in autoimmunity, but contrary to our study, suggest that DC transfers precipitate autoimmune disease. Knight et al., using experimental models of autoimmune disease ( thyroiditis and encephalomyelitis) demonstrated the efficient transfer of disease by splenic DCs derived from animals with active autoimmune disease $(12,13)$. Several differences exist between our experiments and those of Knight, such as the use of a spontaneous autoimmune disease model, the use of draining LN DCs, and footpad versus intravenous injection. The apparent disparity between these experiments and ours may exist because PLN DCs may contain high levels of antigen in comparison to splenic DCs. Knight et al. have suggested that the concentration of antigen present on DC may play a key role in the type of immune response generated by these cells (18). They have demonstrated that high concentrations of antigen on DCs tend to suppress the immune response to the given antigen. In our experiments large numbers of PLN DCs reaching a target $\mathrm{LN}$, the popliteal, may present a high concentration of islet antigen to $\mathrm{T}$ cells and generate a regulatory immune response.

Lymphoid cells, other than DCs, which contaminate DC preparations could contribute to, or may be primarily responsible for protecting NOD recipients from diabetes. $\mathrm{T}$ cells, the major contaminant in DC preparations ( $10-20 \%$ ), could generate protection as $\mathrm{T}$ cells isolated from animals with autoimmune disease can be used as a vaccine to protect recipients (32, 33). Arguing against this possibility is the finding that nylon wool-purified PLN T cells from NOD donors with active autoimmune disease ( $8-12 \mathrm{wk}$ of age ) did not modulate autoimmunity.

In addition accessory cells, such as B cells and macrophages, contained in the PLN DC preparations could present islet antigens, and also stimulate regulatory cells. The number of these cells in DC preparations is small in comparison to the total number of DCs ( $<5 \%$ B cells, and 5-10\% macrophages), but it is nonetheless possible that they could contribute to the modification of the autoimmune response. Indeed, a number of recent studies suggest that B cells and macrophages modify immune, and autoimmune responses.

Recently Eynon and Parker demonstrated in vivo that small resting $B$ cells induce antigen specific $T$ cell tolerance when soluble antigens (Fab fragments of rabbit-anti-mouse IgD) are targeted to these cells (35). B cells contained in the PLN DC populations appear to be of the small, resting type (data not shown). Tolerance in their system, however, is not permanent and lasts only a few weeks. These authors suggest that the mechanism of antigen-specific tolerance induction is through deletion or inactivation of $T$ cells in the periphery by $B$ cells presenting the soluble antigen. It may be that resting $B$ cells in the PLN DC preparation induce anergy or delete autoreactive $T$ cells in recipient NOD mice. Our data, however, suggest that the mechanisms of self tolerance generated by PLN DC transfers is different from that suggested for B cells by Enyon and Parker (35). First, protection provided by PLN DC transfers is long lasting. Second, protection appears to be mediated by cells capable of actively suppressing the transfer of diabetes, which does not fit the model of anergy or clonal deletion. However, the contribution of PLN B cells to the protection phenomena witnessed needs to be more closely evaluated as B cells could augment protection by deletion of autoreactive cells or by inducing anergy.

Further evidence for the modulatory effect of B cells and macrophages comes from the work of Ben-nun and Yossefi in the experimental autoimmune encephalomyelitis (EAE) model (36). These investigators demonstrated that pretreatment of recipients with intraperitoneal injections of gamma interferon-treated macrophages and B cells pulsed with myelin basic protein (MBP) before myelin basic protein and adjuvant immunization, blocked the development of EAE. They suggested that the mechanism of protection provided by MBPpulsed macrophages and B cells is by the diversion of autoreactive cells away from the target organ, the central nervous system.

Our experiments differ in several important ways from those of Ben-nun and Yossefi (36). First, B cells and macrophages were treated with gamma interferon, and the numbers of B cells and macrophages used in these experiments was 100fold greater than the number of these cells contaminating our DC preparations. Our results suggest that the mechanism of protection of PLN DC transfers is not mediated by the diversion of autoreactive cells away from the target organ as suggested by Ben-nun and Yoseffi for macrophages and B cells, but instead is mediated by potent regulatory cells that effectively inhibit the transfer of diabetes. Furthermore, we found no evidence to support the diversion of autoreactive lymphocytes as there was no accumulation of lymphocytes in the footpads of recipients nor was there enlargement of the popliteal LNs of NOD mice that received PLN DC transfers.

However, because of the potential contribution of PLN B cells and macrophages to the potent protective effect of PLN DC transfers it will be important to evaluate the contribution of each of these cell types to this phenomena. We are currently evaluating the effect of the transfer of each of these PLN antigen-presenting cells on the incidence of diabetes in the NOD mouse. It may be that therapeutic strategies using B cells, macrophages, or DCs pulsed with antigens relevant to the autoimmune response will provide viable approaches to the modulation or prevention of clinical autoimmune disease.

Viruses such as murine hepatitis virus are known to modify the incidence of diabetes in NOD colonies (37). The low incidence of diabetes in the PLN DC group could be due to the presence of viruses in these animals that modified disease incidence. However, this is not likely for the following reasons. First, recipients were randomized into the different experimental groups to control for variation in the incidence of diabetes between different litters. This also would control for the vertical transmission of viruses. Second, the different groups of DCs used in the experiments were all derived from the same pool of donor mice. This would control for the transmission of viruses from donor to recipient. All recipient mice were housed under the same environmental conditions as were colony mice. We did not detect a lower incidence of diabetes in colony mice, or in the experimental control groups (i.e., A/I LN DC group) during the experimental observation period. Finally, we found that the NOD mice that received PLN DC transfers that became diabetic were randomly distributed amongst different cages of this experimental group, suggesting that specific cages of PLN DC recipients were not uniquely infected with viruses that lowered diabetes incidence.

These studies demonstrate that the PLN DC transfers are very effective in protecting NOD recipients from diabetes. The 
data also suggest that PLN DC transfers uniquely provide protection from diabetes. This in vivo phenomena may occur because PLN DC carry a biologically relevant islet peptide that is presented to NOD lymphocytes and promotes the activation of regulatory elements. PLN DCs may thus play an important role in an immune regulatory network in the NOD mouse.

\section{Acknowledgments}

The authors wish to thank Dr. Yoko Mullen for her great generosity in the use of her laboratory and animal colony, and Drs. William Clark and Andre Van Herle for their critical review of the manuscript.

Dr. Clare-Salzler is supported by a National Institutes of Health Clinical Investigator Award, U.S. Public Health Service grant DK01861. The UCLA mouse colony is supported through Dr. Mullen's grants from the Nora Eccles Treadwell Foundation, and National Institutes of Health grants DK20827 and DK17980.

\section{References}

1. Makino, S., K. Kunimoto, Y. Muraoka, Y. Mizushima, K. Katagisik, and Y. Tochino. 1980. Breeding of a non-obese diabetic strain mice. Exp. Anim. (Tokyo). 29:1-13.

2. Tochino, Y., T. Kanaya, and S. Makino. 1982. Genetics of NOD mice. In Clinico-patho-genesis of Diabetes Mellitus. G. Mimura, S. Baba, Y. Goto, and J. Kebberling, editors. Excerpta Medica, Amsterdam. 285-291.

3. Fujita, T., R. Yui, Y. Kusumoto, Y. Serizawa, S. Makino, and Y. Tochino. 1982. Lymphocytic insulitis in a "non-obese" diabetic (NOD) strain of mice: an immuno-histochemical and electron microscopic investigation. Biomed. Res. 3:429-436.

4. Wicker, L. S., B. J. Miller, and Y. Mullen. 1984. Transfer of autoimmune diabetes mellitus with spleenocytes from non-obese diabetic (NOD) mice. Diabetes. 35:855-860.

5. Hanafusa, T., R. Yamada, J. Mizagawa, H. Fujino-Kurishara, H. Nakajima, K. Nonaka, and S. Tarui. 1985. Predominance of T lymphocytes in pancreatic islets and spleen of prediabetic non-obese diabetic (NOD) mice: a longitudinal study. Clin. Exp. Immunol. 60:622-630.

6. Sadelain, M. W. J., H. Y. Qin, J. Lanzon, and B. Singh. 1990. Prevention of type 1 diabetes in NOD mice by adjuvant immunotherapy. Diabetes. 39:583589.

7. Oldstone, M. 1990. Viruses as therapeutic agents. I. Treatment of nonobese insulin-dependent diabetic mice with virus prevents insulin-dependent diabetes mellitus while maintaining general immune competence. J. Exp. Med. 171:2077-2089.

8. Serreze, D. V., K. Hamaguchi, and E. H. Leiter. 1989. Immunostimulation circumvents diabetes in NOD/Lt mice. J. Autoimmun. 2:759-776.

9. Satoh, J., T. Seino, S. I. Abo Tomaka, S. Shintani, S. Onta, K. Tamura, T. Sawai, T. Nobunaga, T. Oteki, K. Kumagai, and T. Tozota. 1989. Recombinant human tumor necrosis factor alpha suppresses autoimmune diabetes in nonobese diabetic mice. J. Clin. Invest. 84:1345-1348.

10. Boitard, C., R. Yasunami, M. Daidenne, and J. F. Bach. 1989. T cell-mediated inhibition of the transfer of autoimmune diabetes in NOD mice. J. Exp. Med. 169:1669-1880.

11. Faustman, D., G. Eisenbarth, J. Daley, and J. Breitmeyer. 1989. Abnormal T-lymphocytic subsets in type 1 diabetes. Diabetes. 38:1462-1467.

12. Knight, S. C., J. Farrant, J. Chan, H. Bryant, P. H. Bedford, and C. Bateman. 1988. Induction of autoimmunity with dendritic cells: studies on thyroiditis in mice. 1988. Clin. Immunol. Immunopathol. 48:277-289.

13. Knight, S. C., J. Mertin, A. Stackpoole, and J. Clark. 1983. Induction of immune responses in vivo with small numbers of veiled (dendritic) cells. Proc. Natl. Acad. Sci. USA. 80:6032-6035.

14. Farrant, J., A. E. Bryant, J. Chan, and R. L. Hinsworth. 1986. Thyroglobulin treated blood dendritic cells reduce IgG anti-thyroglobulin antibody in vitro in Hashimoto's thyroiditis. Clin. Immunol. Immunopathol. 41:433-442.

15. Nussenweig, M. C., and R. M. Steinman. 1980. Contribution of dendritic cells to stimulation of the murine syngeneic mixed lymphocyte reaction. J. Exp. Med. 151:1196-1212.

16. Serreze, D. V., and E. Leiter. 1988. Defective activation of T suppressor cell function in non-obese diabetic mice: potential relation to cytokine deficiencies. J. Immunol. 140:3801-3807.

17. Yamashita, U., S. Ono, and H. Hakamura. 1982. The syngeneic mixed leukocyte reaction in mice. II. The I region control of suppressor T cell activity induced in the syngeneic mixed lymphocyte reaction. J. Immunol. 128:10101017.

18. Knight, S. C., R. Hunt, C. Dorse, and P. B. Medewar. 1985. Influence of dendritic cells on tumor growth. Proc. Natl. Acad. Sci. USA. 82:4495-4497.

19. Matzinger, P., and S. Guerder. 1989. Does T-cell tolerance require a dedicated antigen presenting cell? Science (Wash. DC). 338:74-76.

20. Zamoyska, R., H. Waldmann, and P. Matzinger. 1989. Peripheral tolerance mechanisms prevent the development of autoreactive $T$ cells in chimeras grafts with two minor incompatible thymuses. Eur. J. Immunol. 19:111-117.

21. Voorbij, H. A. M., P. H. M. Jeucken, P. J. Kabel, M. De Haan, and H. A. Drexhage. 1989. Dendritic cells and scavenger macrophages in pancreatic islets of prediabetic BB rats. Diabetes. 38:1623-1629.

22. Fujita, T., and R. Yui. 1986. Histologic changes in the pancreatic islets of NOD mice. In Insulitis and Type 1 Diabetes: Lessons from the NOD Mouse. Tarui, Yoshihiro, Tochino, Kyohei, and Nonaka, editors. Academic Press Inc. Orlando, FL. 35-59.

23. Knight, S. C., P. Bedford, and R. Hunt. 1985. The role of dendritic cells in the initiation of immune responses to contact sensitizers. II. Studies in nude mice. Cell. Immunol. 94:435-439.

24. Lens, J. W., and H. A. Drexhage. 1983. A study of cells present in lymph draining from a contact allergic reaction in pigs sensitized to DNFB. Immunology. 46:415-422.

25. Julius, M. H., E. Simpson, and L. A. Herzenberger. 1973. A rapid method for the isolation of functional thymus-derived murine lymphocytes. Eur. J. Immunol. 3:645-649.

26. Miyagawa, J., T. N. Hanafusa, A. Migasaki, K. Yamada, H. Fujino-Kurihara, H. Nakajima, N. Kono, K. Nonaka, T. Yoshihiro, and S. Taurui. 1986. Ultrastructural and immunocytochemical aspects of lymphocytic submandibulitis in the non-obese diabetic (NOD) mouse. Virchows Arch. B Cell Pathol. 51:215-225.

27. Koidi, S. L., K. Inaba, and R. M. Steinman. 1987. Interleukin 1 enhances $T$ dependent immune responses by amplifying the function of dendritic cells. $J$. Exp. Med. 165:515-520.

28. Heufler, C., F. Koch, and G. Schular. 1988. Granulocyte-macrophage colony stimulating factor and interleukin 1 mediate the maturation of murine epidermal Langerhans cells into potent immunostimulatory dendritic cells. $J$. Exp. Med. 167:700-705.

29. Baxtar, A. G., M. A. Adams, and T. E. Mandel. 1989. Comparison of highand low-diabetes incidence NOD mouse strains. Diabetes. 38:1296-1300.

30. Bocheiri, M. H. 1985. T-suppressor clones derived from murine AMLR. Immunology. 56:93-102.

31. Inaba, K., J. P. Metaly, M. T. Crowley, and R. M. Steinman. 1990. Dendritic cells pulsed with protein antigens in vitro can prime antigen specific MHC restricted T cells in situ. J. Exp. Med. 172:631-640.

32. Lohse, A. W., F. Mor, N. Karin, and I. R. Cohen. 1989. Control of experimental autoimmune encephalomyelitis by $\mathrm{T}$ cells responding to activated T cells. Science (Wash. DC). 244:820-822.

33. Maron, R., R. Aerubavel, A. Freidman, and I. R. Cohen. 1983. T lymphocyte line specific for thyroglobulin produces or vaccinates against autoimmune thyroiditis in mice. J. Immunol. 131:2316-2322.

34. Crowley, M., K. Inaba, and R. M. Steinman. 1990. Dendritic cells are the principal cells in mouse spleen bearing immunogenic fragments of foreign proteins. J. Exp. Med. 172:383-386.

35. Enyon, E. E., and D. C. Parker. 1992. Small B cells as antigen presenting cells in the induction of tolerance to soluable protein antigens. J. Exp. Med. 175:131-138.

36. Ben-Nun, A., and S. Yossefi. 1990. Reversal of autoimmune encephalomyelitis by membrane presenting myelin basic protein-associated class II MHC molecule as an approach to immunotherapy of organ-specific autoimmune disease. Eur. J. Immunol. 20:357-361.

37. Wilberz, S., H. J. Partke, F. Dagnaes-Hansen, and L. Herberg. 1991. Persistent MHV (mouse hepatitis virus) infection reduces the incidence of diabetes mellitus in non-obese mice. Diabetologia. 34:2-5. 